\title{
Strategy Control and Directed Forgetting ${ }^{1}$
}

\author{
Walter Reitman, Jane Tanner Malin, Robert A. Bjork And \\ Barbara HigmaN
}

The University of Michigan, Ann Arbor, Michigan 48104

\begin{abstract}
Signals to subjects to forget presignal items (F-items) can completely eliminate the proactive interference postsignal items (R-items) would normally suffer from the F-items. To determine what happens to F-items, a strategy-control procedure was developed to test memory for F-items without destroying forget-signal credibility. The subjects saw short lists of paired associates, some containing a signal to forget presignal pairs. After each list, a single stimulus probe tested memory for the appropriate response. Tested pairs were always R-pairs unless a prearranged cue informed the subjects that the stimulus was from an F-pair. Although F-pairs did not interfere with recall of R-pairs, there was substantial recall of and interference among F-pairs. The results support a set differentiation mechanism of directed forgetting.
\end{abstract}

The processes by which information no longer needed is eliminated or set aside are as fundamental to the efficient functioning of an information processing system as are the processes by which information is acquired. Any limited capacity system without the means to select and eliminate is doomed to an unfortunate and incoherent end; without some mechanism to prevent old information from interfering with the processing of current information, the system's output will eventually bear no sensible relation to its input.

One promising approach to the problem of information elimination is the recent active research on directed forgetting (for reviews see Bjork, 1972; Epstein, 1972). This approach centers on the use of signals to subjects that they can forget some or all of the information

\footnotetext{
${ }^{1}$ We are indebted to J. E. K. Smith for his advice on the design of this study and the analysis of the data, and to Judith Reitman, Donald Norman, and Gordon Bower for a close reading and thoughtful comments on an earlier draft. We also acknowledge support received from the National Institutes of Mental Health, the National Science Foundation, and the Mental Health Reseach Institute of The University of Michigan. Requests for reprints should be sent to Walter Reitman, The University of Michigan, 205 North Washtenaw Place, Ann Arbor, Michigan 48104.
}

they have been presented. Such signals (F-cues) can have remarkable effects; subjects are able, for example, to take advantage of F-cues in some situations in a way that completely eliminates the proactive interference that information presented subsequent to the F-cue would normally suffer from the preceding tobe-forgotten information. The present study concerns itself with the fate of to-be-forgotten information (F-items). Rather than to indirectly investigate the effects of an F-cue on F-items by looking at whether or not F-items interfere with to-be-remembered information (R-items), the present study employs direct tests of subjects' memory for F-items.

The reasons one would want to test subjects' memory for F-items are obvious: The extent to which F-items are or are not recallable or recognizable in different situations provides a basis for inferring the mechanisms by which F-items are marked, segregated, erased, or whatever. Unfortunately, there are obvious and formidable problems in attempting to test F-items. Given that the directed-forgetting paradigm is based on instructing subjects that an F-signal means forget the designated items, any test of F-items violates fair play to some extent. 
Several different procedures have been used to test whether subjects can recall F-items. Weiner and Reed (1969) introduced the straightforward procedure of cuing subjects at the onset of an item in a Brown-Peterson paradigm whether to remember or to forget the item, but testing for recall of the item in either case. This procedure for testing Fitems is, unfortunately, as problematical as it is straightforward. Since subjects are cued to forget items they know they will have to recall, one cannot be sure that they are trying to forget when cued to do so, or, for that matter, that they always try to recall F-items when they are asked to. A second procedure (Bjork, 1970; Bruce \& Papay, 1970; Davis \& Okada, 1971) consists of testing F-item recall on the last trial or two of an experiment, at which time subjects presumably believe F-cues, and, since the experiment proper is over, one can do little harm by destroying their faith in F-cues. There are three problems with this procedure. (a) Since such tests come as a surprise to the subjects, they are disruptive and may, therefore, result in impaired performance. (b) Only one or two such surprise tests can be tacked on the end of a given experiment, which makes them an inefficient way to gather data on the recall of F-items. (c) And because of practice effects, proactive interference, or whatever, performance on trials at the end of an experiment may not be characteristic of performance throughout the experiment. A third procedure (see Woodward \& Bjork, 1971) consists of a delayed recall test for all items presented during an experimental session. The results of such a test can be informative, but they provide no measure of subjects' ability to recall F-items immediately following any given trial of the experiment.

This paper introduces a new procedure for testing F-items that is designed to avoid the problems inherent in the procedures outlined above. The procedure consists of a combination of pretraining and strategy-control instructions that permits the testing of $\mathrm{F}$ items on a number of different trials during an experiment. Subjects are first trained on a series of trials during which F-items are never tested. They are then informed that the remainder of the experiment will consist of trials just like those already presented, except that there will be infrequent trials on which F-items rather than $\mathrm{R}$-items will be tested. They are told that all tests of F-items will be designated by a special signal presented at the time of any such test, and that their best strategy is to behave as if F-items were never going to be tested; that is, they are told to continue, as in the pretraining session, to forget F-items and to remember R-items.

The success of this procedure depends, of course, on subjects behaving in the "as if" fashion asked of them. One purpose of the present experiment is to explore the use of such strategy-control manipulations as experimental tools. Given that subjects can establish a measure of control over their strategies, the systematic control of strategies constitutes an important tool for investigating basic cognitive mechanisms (Reitman, 1970). With the present procedure, if performance on trials when R-items are tested is characteristic of performance in an equivalent experiment not involving tests of F-items, then one has some evidence that subjects were consistently trying to forget F-items.

In order to have a reference experiment against which to evaluate the effects of the strategy-control procedure, the present experiment follows closely an experiment reported by Bjork (1970). Subjects were presented lists of one to eight paired associates, and following each list a stimulus member from one of the pairs in the list was presented as a probe test of subjects' memory for the paired response. In some lists, there was a signal to forget the pairs presented prior to the signal. Since subjects could not anticipate when a list would contain such a signal (F-cue), they were forced to attempt to learn each pair as it was presented. At the end of lists that contained a forget signal, one of the postsignal pairs was tested on all but those infrequent 
trials where asterisks next to the test stimulus informed the subjects that a presignal pair (F-pair) was being tested.

\section{METHOD}

\section{Subjects}

The subjects were 82 undergraduate women in the University of Michigan. They were each paid $\$ 2.00$ for their participation.

\section{Apparatus and Materials}

The apparatus was a PDP-8 computer and scope. Each paired associate in a list was presented for 2.2 $\mathrm{sec}$, with a .8 -sec interval between pairs. The first pair in a list was shown either directly above or below a dashed horizontal reference line on the scope. The subsequent pairs in the list were either all presented in the same position as the first pair, or, at some point in the list, there was a change in position, relative to the reference line, of the next pair in the list. The latter event was a prearranged signal to subjects to forget the pairs in the list presented prior to the position change.

The paired associates consisted of nonsense syllables as stimuli (selected from Archer, 1960, norms range 38-62) and words as responses (ThorndikeLorge rating 18-AA). Following each list, a single test stimulus was presented as a probe test of subjects' memory for the response that had been paired with that stimulus on the list. The test stimulus appeared on the screen by itself, without a reference line, $.8 \mathrm{sec}$ after the last pair in the list and it remained on the screen until the subject wrote her response on a $3 \times 5$ card and placed the card in a box in front of her. When a presignal (F-pair) was tested, an asterisk was presented next to the test stimulus as an indicator to subjects that the word to be recalled was from a pair they had been instructed to forget.

The experimental materials, the timing intervals, and the general procedure were chosen to correspond exactly to Bjork's (1970, Experiment I) reference experiment. The only differences other than the testing of F-items and strategy manipulation in the present study were that Bjork used a Carousel slide projector to present his lists, and a color change in the background of a slide served as the forget cue.

\section{Design}

In what follows, $(a, b)$ denotes a list of $a$ presignal pairs and $b$ postsignal pairs, and $(a, b: c)$ indicates a test of the $c$ th postsignal pair in an $(a, b)$ list. An asterisk is added, giving $\left(a, b:{ }^{*} c\right)$, if the tested trigram came from a presignal pair.

Lists followed by tests of postsignal pairs varied in length from one to eight pairs. Each such list included $0,1,2$, or 3 presignal pairs, followed by $1,2,3,4$, or 5 postsignal pairs. Combining each of the four possible presignal sublist lengths with each of the five possible postsignal sublist lengths resulted in 20 different presignal-postsignal list constructions. Sixty lists were required in order to test every postsignal serial position in every $(a, b)$ list type. The complete set of test conditions is shown in Table 1.

Lists used to test recall of presignal pairs were constructed of $1,2,3$, or 4 presignal pairs, followed by 1,2 , 3 , or 4 postsignal pairs, except that total list length did not exceed five pairs. There were 10 such list constructions: $(1,1),(1,2),(1,3),(1,4),(2,1),(2,2),(2,3),(3,1)$, $(3,2)$, and $(4,1)$. The constraint on total length was adopted to limit the number of tests of presignal pairs. Every presignal serial position was tested once, except in the condition where there were four presignal pairs. Here only the $(4,1: * 4)$ test was included, again in order to minimize tests of pairs subjects had been asked to forget. Altogether, 17 tests of presignal pairs were included in the experiment. The set of presignal test conditions is shown in Table 2.

One hundred and fourteen lists of trigram-word pairs were used in the experiment. Five of the lists were presented as examples during the instructions. Sixty of the remaining 105 lists were identical to the 60 used in Bjork's (1970) study, and the other 49 lists were constructed from trigrams and words similar to those used by Bjork.

After subjects had been practiced in the standard experimental procedure used by Bjork (1970), they worked through 16 lists analogous to the first 16 of the 60 lists from Bjork's Experiment I, to stabilize their expectations and performance. Next, after they had been given the additional strategy control instructions, the 93 lists constituting the experiment proper were presented. The 93 lists consisted of a replication of Bjork's 60 lists intermixed with 17 lists testing recall of presignal pairs, plus a final 16 lists. Three of the 16 final lists were followed by uninformed tests of presignal pairs (no asterisk appeared along with the test stimulus); the remaining lists were included for other purposes and are not further discussed in this paper. An adaptation of the counterbalancing procedures described by Bjork was used to generate the 16 different sets of 109 lists. This procedure was employed in order to minimize effects due to individual pairs and to replicate Bjork's (1970) design.

\section{Procedure}

After the initial instructions, practice lists, and 16 training lists, the experimenter read the additional strategy control instructions describing the "as if" procedure the subject was to follow. The significant paragraphs are here reproduced verbatim:

We are also interested in what happens to the old pairs that were forgotten, so occasionally in the 
next lists we will check on a pair that you were told to forget about. You will always know when this happens, because the nonsense syllable will have an asterisk in front of it. Let me show you an example. [An additional example list was presented.]

If you happened to remember it, the word you would have written down was knight. Okay? The asterisk was the signal that the pair was one you were supposed to forget about. In the rest of the experiment there will be a few asterisked items. Their purpose is to let us compare what happens to pairs you are trying to remember with what happens to pairs you are no longer trying to keep in mind. If an asterisked nonsense syllable occurs and you remember the word that goes with it, fine; write it down. The important thing, though, is to keep on doing just what you have been doing up until now. When there is a change in position from above the line to below, or vice versa, forget about the old pairs and try to remember the new ones. Any questions?

Immediately upon completion of the experiment, each subject was interviewed to determine whether she had been able to carry out the strategy control instructions successfully. She was asked what she thought the purpose of the experiment was; whether she had had any doubts about what we were looking for; whether she had been distracted by the occasional tests of pairs she was supposed to forget; whether those tests had changed her performance in any way. She was also asked what she had done to memorize pairs, to forget presignal pairs, and to respond to the test syllables.

\section{RESUlTS}

There was no prior basis for deciding whether occasional failure to follow the strategy control instructions might affect performance significantly. Thus, only those subjects whose answers suggested that they had followed the instructions perfectly, with no evidence of lapses at any time during the course of the experiment, were included in the

TABLE 1

Proportions Correct on Tests of Postsignal Pairs

\begin{tabular}{|c|c|c|c|c|c|}
\hline \multirow{2}{*}{$\begin{array}{c}\text { Number of } \\
\text { postsignal pairs }\end{array}$} & \multirow{2}{*}{$\begin{array}{l}\text { Postsignal } \\
\text { pair tested }\end{array}$} & \multicolumn{4}{|c|}{ Number of presignal pairs } \\
\hline & & 0 & 1 & 2 & 3 \\
\hline 1 & (1) & .97 & 1.00 & .97 & 1.00 \\
\hline \multirow{3}{*}{2} & (1) & .94 & .77 & .84 & .84 \\
\hline & (2) & 1.00 & .97 & .97 & .88 \\
\hline & Mean & .97 & .87 & .91 & .86 \\
\hline \multirow[t]{4}{*}{3} & (1) & .59 & .50 & .66 & .56 \\
\hline & (2) & .66 & .72 & .75 & .75 \\
\hline & (3) & .87 & .91 & .97 & .91 \\
\hline & Mean & .71 & .71 & .79 & .74 \\
\hline \multirow[t]{5}{*}{4} & (1) & .41 & .50 & .41 & .34 \\
\hline & (2) & .47 & .50 & .50 & .38 \\
\hline & (3) & .69 & .66 & .69 & .50 \\
\hline & (4) & .94 & .88 & .94 & .84 \\
\hline & Mean & .63 & .63 & .63 &.$\underline{.52}$ \\
\hline \multirow[t]{6}{*}{5} & (1) & .34 & .28 & .34 & .47 \\
\hline & (2) & .28 & .28 & .34 & .22 \\
\hline & (3) & .31 & .47 & .40 & .28 \\
\hline & (4) & .59 & .47 & .59 & .44 \\
\hline & (5) & .94 & .78 & .91 & .91 \\
\hline & Mean & .49 & .46 & .52 & .46 \\
\hline Grand mean & & .75 & .73 & .76 & .72 \\
\hline \multicolumn{2}{|c|}{ Mean from Bjork's Experiment I } & .73 & .75 & .77 & .76 \\
\hline
\end{tabular}


basic experimental group. Eighty-two subjects were tested in order to secure a basic experimental group of 32 subjects, with two subjects assigned to each of the 16 sets of lists.

The experiment does not require that all subjects be able to follow the instructions, but it entails predictions only for those who do. Thus, the following analyses concentrate primarily on the basic experimental group.

\section{Recall of Postsignal Pairs}

Table 1 presents the proportion of correct responses for the 32 criterial subjects for every combination of list type and serial position tested in the 60 replication lists. These results are strikingly similar to those obtained by Bjork (1970). In particular, the presignal pairs appear to provide no proactive interference: Performance in the cases where the length of the postsignal sublist is held constant is independent of the number of presignal pairs in the list. The effect of serial position within postsignal sublists also appears in every way typical of the results obtained in such probe experiments.

Whereas Table 1 implies that presignal items do not interfere proactively with subjects' performance on postsignal items, Figure 1 shows that postsignal items preceding a tested item do produce substantial proactive interference. At each level of retroactive interference, as defined by the numbers of pairs presented

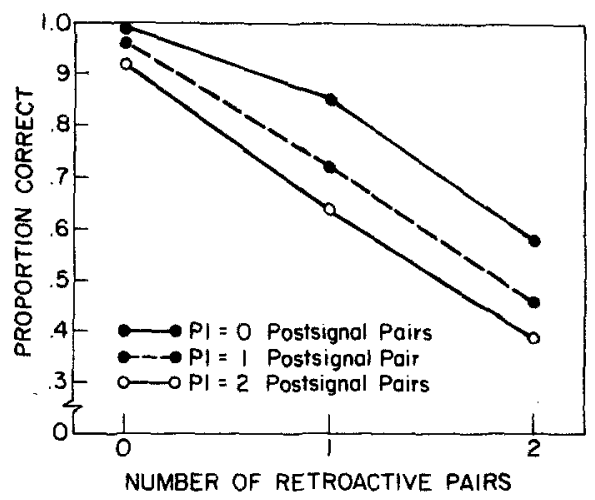

FIG. 1. Proactive interference (PI) effect of postsignal pairs upon recall of subsequent postsignal pairs, averaged over $0,1,2$, and 3 presignal pairs. after the probed pair, performance on postsignal pairs declines as a function of the number of preceding postsignal pairs ( $P I=0,1,2)$.

Further evidence that the instruction to forget attenuates or eliminates proactive interference comes from an examination of intrusions. If we consider only lists in which both presignal and postsignal intrusion errors are possible (i.e., exclude lists in which there were either no presignal items or only one postsignal item), there are a total of 501 errors (intrusions and omissions) in all. Of these, .05. were presignal intrusions, and .82 were postsignal intrusions. In Bjork's (1970) experiment (see his Table 2), the comparable proportions. were .04 and .66 . Thus, the ratio of presignal to postsignal intrusions is $1: 16$ in both cases.

\section{Recall of Presignal Pairs}

The preceding analysis of performance on postsignal pairs yields a very pleasant result: Despite the added strategy control procedures, the probes of presignal pairs, and the change in apparatus, the instruction to forget appears to have eliminated proactive interference due to presignal pairs exactly as in Bjork's (1970) experiment. Thus, one can analyze performance on presignal pairs with some confidence that at least the 32 criterial subjects were consistently trying to forget the presignal pairs. The unparenthesized numbers in Table 2 give the proportions of correct responses to probes of presignal pairs for each of the $17\left(a, b:{ }^{*} c\right)$ list constructions used in the experiment. It is clear from Table 2 that considerable information about these pairs is retained. However, the table also shows an increased rate of information loss over time due to the instruction to forget. The parenthesized numbers are from tests of the same serial positions in lists having comparable total lengths, but no signals to forget. If we compare corresponding figures, contrasting for example the .94 proportion correct for the $(0,2: 1)$ lists with the .75 proportion correct for the $(1,1: * 1)$ lists, we see that performance on pairs from lists without a signal to forget always is at least as good as 
TABLE 2

Proportions Correct on Tests of Presignal Pairs

\begin{tabular}{cccccc}
\hline \multirow{2}{*}{$\begin{array}{c}\text { Number of } \\
\text { presignal pairs }\end{array}$} & $\begin{array}{c}\text { Presignal } \\
\text { pair tested }\end{array}$ & \multicolumn{4}{c}{ Number of postsignal pairs } \\
\cline { 4 - 7 } & $\left(^{*} 1\right)$ & $.75(.94)$ & $.34(.59)$ & $.19(.41)$ & $.09(.34)$ \\
2 & $\left(^{*} 1\right)$ & $.44(.59)$ & $.22(.41)$ & $.06(.34)$ & \\
& $(* 2)$ & $.63(.66)$ & $.34(.47)$ & $.17(.28)$ & \\
& $\left({ }^{*} 1\right)$ & $.28(.41)$ & $.25(.34)$ & & \\
& $\left({ }^{*} 2\right)$ & $.34(.47)$ & $.28(.28)$ & & \\
& $(* 3)$ & $.34(.69)$ & $.13(.31)$ & & \\
\hline
\end{tabular}

Note. The proportions in parentheses indicate performance on tests of comparable items in lists containing no instruction to forget.

performance on the corresponding presignal pairs, and usually is substantially better.

Table 1 demonstrates that there is no proactive interference effect of presignal items (F-pairs) upon postsignal items (R-pairs). Presignal items, however, do have a substantial proactive interference effect upon subsequent presignal items, as is evident from Figure 2. The correct recall proportions for presignal pairs shown in the figure are averaged over constructions in which the presignal pairs are followed by either one or two postsignal pairs. ${ }^{2}$ For instance, the leftmost point in the figure, with a value of .27 , is an average for the $(3,1: * 1)$ and $(3,2: * 1)$ constructions.

Comparing corresponding points, we find, for example, the correct recall proportion for pairs immediately preceding the signal to forget to be .54 when the presignal sublist contains only that one pair; it is .49 when the tested pair is preceded by one other presignal pair; and it is only .24 when the tested pair is preceded by the two other presignal pairs. These are the three vertically aligned points above * $(n)$ in Figure 2.

Intrusions on tests of presignal pairs reveal

${ }^{2}$ Since the total length of the lists used to test presignal recall could not exceed five pairs, postsignal sublists containing more than two pairs could not occur with all possible presignal sublists. Hence lists containing postsignal sublists of three or four pairs were not considered in this analysis.

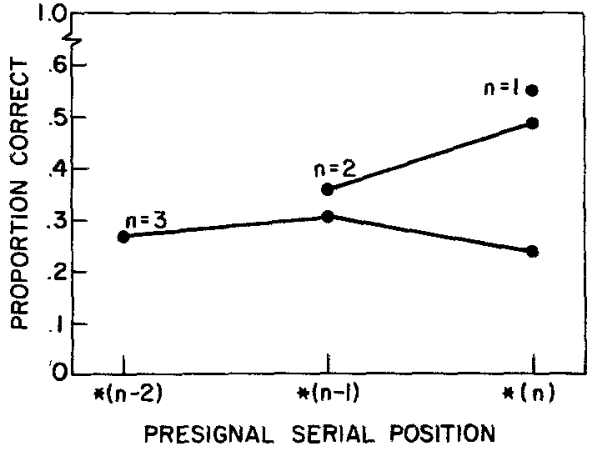

Fig. 2. Proactive interference effect of presignal pairs upon recall of subsequent presignal pairs, where $n$ denotes the total number of presignal pairs. *( ) denotes the position tested, with * $(n)$ being the final presignal pair.

a very different pattern from that on tests of postsignal pairs, reported above. On tests of presignal pairs, averaged over all cases in which both kinds of intrusions are possible, the proportion of presignal intrusions is .30 , and the proportion of postsignal intrusions is .11 . This is in clear contrast to the results for post-signal pairs.

As a further check on the comparability of the procedures used in the present study with those employed by Bjork (1970), three uninformed tests of presignal pairs were included at the end of the present study. The lists corresponded to the $(2,3: * 2),(3,2: * 3)$, and $(3,2: * 1)$ constructions, except that in each case no asterisk was presented with the test 
stimulus. As expected on the basis of Bjork's results, the correct response proportions obtained were all close to zero, $.06, .12$, and .09 , respectively.

\section{Is Memory Capacity Constant?}

Relative to the performance levels obtained when no forget signal is present, we have observed, on the one hand, a decrement in performance on presignal items, and on the other hand, a gain in performance on the postsignal test lists. Taken together, these two observations suggest that the memory capacity a subject uses in this experiment may perhaps be a constant quantity, something allocated over presignal and postsignal items as a function of the instructions to forget.

We can evaluate this conjecture by comparing performance on lists having the same total length, but differing in the number of presignal versus postsignal pairs they contain. Thus, for example, as shown in Table 3, we find that the proportion correct averaged over the $(0,5: 1)$, $(0,5: 2),(0,5: 3),(0,5: 4)$, and $(0,5: 5)$ constructions is .49 . This figure is essentially the same as that obtained when we take comparable averages from all other sets of lists having a total length of five, regardless of the number of presignal pairs those sets contain. Thus, for instance, when we average over the $(3,2: * 1)$, $(3,2: * 2),(3,2: * 3),(3,2: 1)$, and $(3,2: 2)$ constructions, that is, over all lists of length five consisting of three presignal pairs and two postsignal pairs, we find the overall proportion correct to be .48 . The conjecture is not fully supported by the data shown in Table 3 . There is some decrement as the number of presignal pairs increases when we consider all lists of length two, and also in the case of lists of length four. All the figures seem close enough to make the conjecture worthy of further investigation, however.

\section{Performance of Noncriterial Subjects}

All of the analyses described above also were carried out on the data for the 50 noncriterial subjects. The results generally correspond very
TABLE 3

Average Proportion Correct as a Function of Lengths of Presignal and Postsignal Sublists

\begin{tabular}{ccccc}
\hline \multirow{2}{*}{$\begin{array}{c}\text { Total list } \\
\text { length }\end{array}$} & \multicolumn{5}{c}{ Number of presignal pairs } \\
\cline { 2 - 5 } & 0 & 1 & 2 & 3 \\
\hline 2 & .97 & .88 & - & - \\
3 & .71 & .69 & .68 & - \\
4 & .63 & .58 & .60 & .49 \\
5 & .49 & .52 & .51 & .48 \\
\hline
\end{tabular}

Note. Proportions are averages over all serial positions.

closely to the findings already reported for the basic experimental group, but there are some small differences. For example, noncriterial subjects have somewhat higher recall scores for presignal pairs, and they intrude a slightly higher proportion of presignal responses on tests of postsignal pairs. As in these cases, what differences there are between the two groups generally can be accounted for by assuming that noncriterial subjects were less consistent in following the strategy control instructions.

\section{Discussion}

The present experiment was productive in several ways. (a) The results go well beyond any past efforts to test presignal, F-items in terms of providing a picture of the fate of $\mathrm{F}$ items in memory. (b) The results provide strong support for set differentiation as a mechanism of directed forgetting, and they argue against erasure and decay mechanisms. (c) And the results demonstrate the usefulness of strategy control as an experimental technique.

\section{The Fate of To-be-forgotten Items}

The systematic testing of F-items in the current experiment yields a number of important results not available in past studies. (a) There is a stunning difference between performance on informed tests of F-items and uninformed tests of $\mathrm{F}$-items. When a prearranged cue informed subjects that a presignal pair was being tested, they were able to recall the correct response at a 
level equal to about $60 \%$ the level at which they could recall the correct response from a comparable postsignal pair; when an F-pair was tested without informing subjects as to the nature of the test, performance was essentially zero. $(b)$ Even though F-items do not interfere with performance on R-items, they interfere with performance on other $F$ items. As the number of F-pairs increases, performance on any one F-pair decreases systematically although R-pair recall is unaffected. (c) The loss rate of F-item recall over time is very rapid, and can be interpreted as providing a measure of the rate at which unrehearsed items are lost from short-term memory. When a single F-pair was presented and then tested after one, two, three, or four R-pairs had been presented, the probability of correct recall was $.75, .34, .19$, and .09 , respectively. (d) The retroactive interference attributable to a single R-pair on the recall of an F-pair exceeds the retroactive interference attributable to a comparable F-pair on the recall of a preceding Fpair. On the average, each additional R-pair reduces F-pair performance by approximately $50 \%$; each additional F-pair reduces performance on a preceding $\mathrm{F}$-pair by approximately $30 \%$. (e) The data permit an analysis of whether the overall effect of a signal to forget is positive or negative, that is, whether the benefits accruing to $\mathrm{R}$-items are greater or less than the impairment in the recall of F-items. The outcome of the analysis (Table 3 ) is not conclusive, but it appears that the positive and negative effects of a signal to forget are approximately equal in magnitude. $(f)$ Finally, the pattern of intrusions of responses from Rpairs and F-pairs other than the pair tested varies in a striking way with whether the pair tested is an R-pair or F-pair. In general, intrusions are predominantly responses that are appropriate to the set being tested.

\section{Mechanisms of Directed Forgetting}

The results of the present experiment are in strong agreement with an implication derivable from the results of several other studies of directed forgetting (e.g., Block, 1971; Elmes, Adams, \& Roediger, 1970; Woodward \& Bjork, 1971): The lack of proactive interference by presignal pairs on retention of postsignal pairs cannot be accounted for by assuming that presignal items are either erased or decay rapidly from memory. To-be-forgotten items are simply not absent from memory: That substantial information about F-items can be retrieved from memory is demonstrated by the current study, and the studies by Elmes $e t$ al. (1970) and Block (1971) reveal that recognition of F-items in some situations may suffer no impairment at all compared to recognition of R-items.

At the same time that the present results rule out erasure or decay as the sole or principal process by which subjects accomplish the functional forgetting of F-items, they strongly support the set differentiation mechanism proposed by Bjork $(1970,1972)$ in his theory of directed forgetting. Bjork argues that subjects take advantage of a forget instruction in the following way: They differentially group all items following a forget signal into a set that functionally segregates them from the to-beforgotten items, and they devote all rehearsal and mnemonic efforts following the forget signal to the to-be-remembered items. Recall of R-items is assumed not to suffer interference from F-items because retrieval activities are restricted to the set of R-items in memory; since F-items are differentiated in memory by virtue of their being disjoint from the set of $R$ items, they do not interfere.

The following results from the present study are evidence in support of the set differentiation mechanism. (a) On tests of R-pairs, subjects' overt intrusions are almost exclusively responses from other R-pairs. On informed tests of F-pairs, however, intrusions of responses from other F-pairs become several times more frequent than intrusions of responses from R-pairs. Thus, it appears that subjects are able to direct their retrieval efforts to the set of pairs in memory appropriate to the nature of the pair being tested. (b) Further 
evidence for such a differential search is provided by the results of the uninformed tests of F-pairs. Such tests lead subjects to misdirect their retrieval efforts and they retrieve the correct response with negligible frequency. (c) Although F-pairs do not interfere with Rpairs, they interfere with each other. It is as though interference within the forget set occurs in memory, but the forget set does not interfere with the remember set in memory.

Although the results of the present study demonstrate that the absence of proactive interference owing to presignal pairs on the recall of postsignal pairs depends little if at all on the "forgetting" of presignal items, at least in the usual sense of the term, they do not cast much additional light on the mechanism of set differentiation itself. It may be that subjects avoid rehearsing presignal items. Presignal pairs still are available, however, and they somehow must be distinguished from the items to be rehearsed. Since the time interval between the last presignal item and the first postsignal item is no longer than the interval between other pairs of items, it is unlikely that the two sets of items can be differentiated solely on the basis of some kind of time tag. Certainly there is nothing about the items themselves that differentiates presignal from postsignal sets. On the contrary, the items in the two sets are highly similar, and normally we would expect substantial interference, in the form of confusion or miscoding of postsignal items, owing to the context created by the presignal set. The present data would appear to require the conclusion that subjects are capable not only of complex encodings of stimuli (Wickens, 1970), but also of adding new dimensions in the course of encoding.

Although the interviews conducted after the experimental sessions were mainly for the purpose of defining a basic experimental group, subjects also were asked about what they had done to forget presignal pairs when the signal to forget occurred. The answers they gave are at most suggestive, but they do bear in interesting ways on the set differentiation problem. Many of the answers stressed a passive letting go. One typical subject reported that forgetting presignal pairs simply meant not repeating them to herself anymore. Another replied that she just concentrated on the second part. Many described starting over with the postsignal items "as if there were two completely different lists."

There were other kinds of responses as well, however. One subject tried to deal with each list by forming a single sentence from its pairs. When the signal occurred, she responded by "starting a new sentence." Another, in addition to whatever mental operations she carried out, reported that she switched fingers whenever the position of the pairs changed with respect to the line. The relation of these statements to the psychological processes involved is problematic. Still one cannot help being struck by the sheer variety in the subjects' reports. If taken seriously, they suggest that set differentiation may best be regarded as a functional concept, an end result achievable by any of a variety of strategies and techniques.

This study was designed to provide data about the techniques subjects use for organizing information, and in particular, about how information no longer needed is disposed of. In that light, the conclusion that subjects dispose of information by marking it, that is, by adding information during encoding, may seem somewhat paradoxical. One might note, however, that such procedures are quite routine in dealing with comparable information handling problems in computer systems. Furthermore, for what they are worth, the subjects' comments cited above are entirely compatible with such a conjecture.

\section{Strategy Control as an Experimental Technique}

The findings reported in the results section of this paper indicate that the strategy control training achieved its purpose. It is true that only 32 of the 82 subjects satisfied the interview criterion. But all subjects who gave any indication at all that they had not followed the instructions perfectly throughout the entire 
experiment were excluded. Many of those excluded reported nothing more than a difficulty in carrying out the instructions on the first two or three trials, or a very infrequent lapse over the course of the experiment. Thus, the large number of noncriterial subjects mainly reflects the stringency of the criterion. The fact that the results for the noncriterial subjects generally coincide closely with those for the basic experimental group suggests that most subjects are capable of the kind of strategy control required in this study.

It is interesting to note that the original Bjork paradigm itself assumes that subjects can be instructed to exert a degree of voluntary control ("forgetting" or "not forgetting") over their mental activity. Thus Bjork's studies, like much other current work, for example the extensive current work on imagery, reffect the gradual alteration in our notions of reasonable experimental procedure that has resulted from the growth of strategy system conceptions of behavior. In that respect, the present experiment only goes a step further. It assumes that humans are capable of developing and utilizing complex strategies, it encourages development of the desired strategies through appropriate instructions and training, and it compares resulting performance with performance in a reference condition, to assess the adequacy of the experimental procedures directly. If we do not control strategy by training and instruction, some subjects will use imagery, others will make up stories, and still others, noting that the experimenter said nothing about mnemonics, will avoid using them even if they know about them. What we accomplish by not controlling strategies is to limit the information we get from our data.

Strategy control runs counter to some standard statistical modes of thought in that it depends upon an unwillingness to treat strategy change as a source of error variance. But as Reitman (1970) points out, those modes of thought are themselves inconsistent with an information processing conception of beha- vior. Behavior is not to be accounted for in terms of a set of additive components. It is generated by a system in which some of the components (the strategies) switch other components on and off at various times and as a function of the outcome of prior processing. In this light strategy control procedures, with suitable checks, are the appropriate experimental too!s.

\section{REFERENCES}

ARCHER, E. J. A reevaluation of the meaningfulness of all possible CVC trigrams. Psychological Monographs, 1960, 74 (No. 10, Whole No. 497).

BJork, R. A. Positive forgetting: The noninterference of items intentionally forgotten. Journal of Verbal Learning and Verbal Behavior, 1970, 9, 255-268.

BJoRK, R. A. Theoretical implications of directed forgetting. In A. W. Melton \& E. Martin (Eds.) Coding processes in human memory. Washington, D.C.: Winston, 1972.

BLOCK, R. A. Effects of instructions to forget in shortterm memory. Journal of Experimental Psychology, 1971, 89, 1-9.

Bruce, D., \& Papay, J. P. Primacy effect in single-trial free recall. Journal of Verbal Learning and Verbal Behavior, 1970, 9, 473-486.

DAVIS, J. C., \& OKADA, R. Recognition and recall of positively-forgotten items. Journal of Experimental Psychology, 1971, 89, 181-186.

Elmes, D. G., Adams, C. A., \& Roediger, H. L. Cued forgetting in short-term memory: Response selection. Journal of Experimental Psychology, 1970, 86, 103-107.

Epstein, W. Mechanisms of directed forgetting. In G. H. Bower (Ed.) The psychology of learning and motivation, Vol. 6. New York: Academic Press, 1972.

ReITMAN, W. What does it take to remember? In D. A. Norman (Ed.) Models of human memory. New York: Academic Press, 1970.

WEINER, B., \& REFD, H. Effects of the instructional sets to remember and to forget on short-term retention: Studies of rehearsal control and retrieval inhibition (repression). Journal of Experimental Psycho$\log y, 1969,79,226-232$.

WICKENS, D. D. Encoding categories of words: An empirical approach to meaning. Psychological Review, 1970, 77, 1-15.

WoOdwARd, A. E., \& BJoRk, R. A. Forgetting and remembering in free recall: Intentional and unintentional. Journal of Experimental Psychology, 1971, 89, 109-116.

(Received August 8, 1972) 